\title{
Determinantes da Confiança do Consumidor e Dinâmica da Política Monetária no Brasil
}

\author{
Determinants of Consumer Confidence and \\ Monetary Policy Dynamics in Brazil
}

Rafaela Maiara Caetano*
Cleomar Gomes da Silva ${ }^{\dagger}$

\begin{abstract}
Resumo
O objetivo deste artigo é investigar os determinantes da confiança do consumidor e impactos na dinâmica da política monetária no Brasil. A metodologia econométrica aplicada envolve modelos Autorregressivos de Defasagem Distribuída (ARDL) aliados à abordagem de cointegração. Para dados entre janeiro de 2003 e dezembro de 2016, os resultados sugerem a existência de uma relação de longo prazo entre o índice de confiança do consumidor e as demais variáveis analisadas. Em relação à dinâmica de curto prazo, o mecanismo de correção de erros varia entre $1,9 \%$ e $8,7 \%$, a depender do modelo estimado. Isto sugere que variáveis econômicas influenciam a confiança do consumidor e, quando esta confiança é posta em xeque, ela se recupera de forma lenta.
\end{abstract}

Palavras-Chave: Confiança do Consumidor; Política Monetária; ARDL; Cointegração.

\begin{abstract}
The aim of this article is to investigate the determinants of consumer confidence in Brazil and possible impacts on monetary policy actions. The econometric methodology applied is based on Autoregressive Distributed Lag (ARDL) Models, particularly the Bounds Testing (ARDL) Approach to Cointegration. For monthly data between January 2003 and December 2016, the empirical results suggest that there is a long run relationship between consumer confidence and the other variables analyzed. As for short-run dynamics, the error correction mechanism varies between $1.9 \%$ and $8.7 \%$, depending on the estimated model. This suggests that economic variables influence consumer confidence, and when there is a break in confidence, its recovery is very slow.
\end{abstract}

Keywords: Consumer Confidence, Monetary Policy, ARDL, Cointegration JEL Classification: C22; E52; E71.

\footnotetext{
"Doutoranda pela Universidade Federal de Lavras. Mestre em Economia pela Universidade Federal de Uberlândia. E-mail: rafaellamaiara@hotmail.com

+ Universidade Federal de Uberlândia (IERI-UFU), Programa de Pós-Graduação em Economia \& Pesquisador Associado do CNPq. E-mail: cleomargomes@ufu.br. O autor agradece o apoio financeiro do CNPq, CAPES e FAPEMIG.
} 


\section{Introdução}

É cada vez mais visível a forma como fatores emocionais e psicológicos influenciam tomadas de decisões dos agentes econômicos. Isso faz com que indicadores de confiança tenham ganhado importância analítica nas últimas décadas, por mensurarem justamente esses fatores qualitativos. Por terem ágil divulgação, estes indicadores se tornam úteis na antecipação de comportamentos de variáveis macroeconômicas. Assim, entender os determinantes da confiança do consumidor contribui para melhorar a qualidade e a efetividade da elaboração dos vários tipos de políticas econômicas.

Do ponto de vista da política monetária, suas decisões são fundamentalmente baseadas no comportamento do consumidor, uma vez que são amparadas pela dinâmica de um índice de preço ao consumidor e pelo comportamento da demanda como um todo. Desta forma, analisar os determinantes da confiança do consumidor se torna um questionamento essencial. Esta discussão não é menos importante no Brasil, dado que a capacidade de se determinar quais as variáveis mais importantes e de maior impacto sobre a confiança do consumidor dá aos formuladores de política econômica a oportunidade de tomadas de decisões mais acuradas e com menor custo de bemestar social.

O objetivo deste trabalho é investigar os determinantes da confiança do consumidor no Brasil, assim como os possíveis efeitos na dinâmica da política monetária brasileira. A hipótese deste estudo se baseia no fato de que o índice de confiança do consumidor brasileiro pode exercer influências sobre a atuação da política monetária por meio do canal das expectativas, impactando o lado real da economia. A metodologia econométrica aplicada envolve modelos Autorregressivos de Defasagem Distribuída (ARDL) aliados à abordagem de cointegração, para dados entre janeiro de 2003 e dezembro de 2016.

O trabalho encontra-se estruturado em quatro seções, além desta introdução. A primeira destina-se a uma revisão teórica e empírica da literatura pertinente ao tema. Na segunda retratam-se a metodologia econométrica e os dados utilizados na pesquisa. A terceira seção apresenta e discute os resultados das estimações econométricas. Por fim, têm-se as considerações finais. 


\section{Revisão de literatura}

\subsection{A confiança do consumidor e sua capacidade preditiva}

A literatura sobre a confiança do consumidor é bastante extensa, principalmente nos estudos que analisam a sua capacidade preditiva. Em menor número estão os estudos voltados para investigar os determinantes da confiança do consumidor, especialmente para o caso do Brasil.

Debes et al. (2014) salientam que existem duas visões estruturais alternativas sobre a confiança do consumidor e seus impactos sobre a economia. A primeira delas é denominada de "news approach", em que consumidores estão expostos a informações sobre os fundamentos futuros da economia e, dada a racionalidade dos agentes, a tendência é que os efeitos sejam duradouros sobre a atividade econômica quando eles levam em conta tais "notícias". A segunda visão é denominada de "pure sentiment" ou "animal spirits", introduzida por Pigou (1929) e Keynes (1936). A ideia se baseia no fato de que surtos de otimismo ou pessimismo podem amplificar o ciclo econômico, podendo ser autônomos e desvinculados dos fundamentos futuros, o que se torna quantitativamente importantes para as flutuações dos ciclos de negócios. De fato, Keynes (1936) salienta que os denominados "animal spirits" podem influenciar a economia real na medida em que as expectativas desses agentes sobre os acontecimentos impactam direta ou indiretamente as variáveis macroeconômicas.

Do ponto de vista empírico, Katona (1968) foi um dos pioneiros na análise dos impactos da confiança do consumidor, salientando que os consumidores não reagem apenas às mudanças nas variáveis macroeconômicas, mas também aos fatores não econômicos, como eventos sociais ou crises políticas. Posteriormente, houve intensificação em torno de esforços para melhor compreensão, principalmente aqueles relacionados à capacidade preditiva. Dentre eles estão os estudos de Acemoglu e Scott (1994), Carroll et al. (1994), Ludvigson (2004) e Kwan e Cotsomitis (2006). Também podem ser citados Belessiotis (1996), para a França; Dees e Brinca (2013), para os EUA e Zona do Euro; Fan e Wong (1998), para Hong Kong; Paradiso et al. (2014), para o caso italiano, além de Çelik e Öserkek (2009) para União Europeia, dentre outros.

Para o caso do Brasil, Lopes e Jesus (2016) investigaram a capacidade preditiva do índice de confiança do consumidor sobre o consumo doméstico no Brasil. Os autores utilizaram dados trimestrais de 1997 a 2015 e encontraram uma 
relação positiva entre taxa de crescimento do PIB e crescimento do índice. Por outro lado, o estudo não forneceu evidências que assegurassem um relacionamento entre o crescimento da taxa de juros e a taxa de crescimento do consumo.

De Mello e Figueiredo (2014) também analisaram a capacidade preditiva dos principais índices de confiança disponíveis no Brasil para o período de 2002 a 2014, utilizando a abordagem VAR. Os resultados sugeriram que o índice de confiança da indústria é mais importante na previsão das variáveis de atividade econômica, tanto presentes quanto futuras, do que os outros índices utilizados, como o do consumidor.

Akerlof e Shiller (2010) argumentam que a psicologia humana conduz eventos financeiros em todo mundo, e que o termo confiança aparece com frequência na literatura de negócios, mas eles enfatizam outros elementos além da capacidade preditiva da confiança. Em meio à crise econômica de 2008, verificouse uma ausência de confiança generalizada entre os consumidores, fazendo com que as políticas econômicas que se julgavam exitosas fossem prejudicadas. Ademais, ainda de acordo com os autores, a restauração da confiança se torna fundamental para a recuperação econômica, especialmente em períodos de recessões, como ocorreu na Grande Depressão e após a Segunda Guerra Mundial.

O relacionamento entre comportamento do consumidor e política monetária pode ser visto no trabalho de Silvia e Iqbal (2011), em que forneceram um quadro teórico destacando o papel da confiança nos ciclos econômicos, assim como os efeitos das políticas monetária e fiscal sobre a confiança para os EUA. Além disso, os autores debateram como as políticas monetária e fiscal restauram a confiança. Os autores estimaram um Vetor Autorregressivo (VAR) para o período de 1959 a 2009 (utilizando dados mensais e trimestrais) e os resultados sugeriram que as políticas monetária e fiscal têm uma relação estável de curto e longo prazos com a confiança e Produto Interno Bruto Real (PIB real).

Lapp e Pearce (2012), por sua vez, examinaram se notícias econômicas afetam diretamente as mudanças de política monetária, mensuradas por mudanças nos preços futuros da taxa de fundos federais, também nos EUA. Os autores utilizaram dados de alta frequência (diários), para o período de 1995 a 2008, e encontraram evidências de que: i) notícias econômicas movem os preços dos ativos e que o mercado futuro dos fundos federais reage lentamente com algumas notícias, em especial a notícias de inflação; ii) diversos eventos de notícias têm pouco ou 
nenhum efeito sobre a política monetária esperada, demostrando que preços dos ativos serão afetados por um canal diferente do que o de mudança nesta política.

\subsection{Determinantes da confiança do consumidor}

Quanto aos estudos destinados à investigação dos determinantes da confiança do consumidor, pode-se dizer que estes consideram um grande grupo de variáveis. De maneira geral, esses determinantes podem ser coletados em três grandes categorias, quais sejam, variáveis macroeconômicas, variáveis financeiras e outros fatores. As variáveis macroeconômicas são utilizadas para mensurar a taxa de crescimento, como a produção industrial, renda disponível, produto interno bruto, taxa de desemprego, dentre outras. Em termos de variáveis financeiras, são utilizados índices de ações, indicadores de riqueza, taxa de câmbio e etc. Por fim, os outros fatores são representados principalmente por fatores políticos ou outras variáveis exógenas de interesse. Embora a listagem de variáveis nas duas primeiras categorias possa manter um certo padrão entre os estudos, elas podem sofrer variações nos diferentes países dadas suas especificidades (Gurgür e Kilinç, 2015).

Quanto às variáveis macroeconômicas e financeiras, Delorme et al. (2001) analisaram os determinantes da confiança do consumidor no Reino Unido por meio das seguintes variáveis: inflação, taxa de juros real, riqueza habitacional, riqueza financeira e taxa de desemprego no consumidor. Os resultados mostram que apenas a taxa de desemprego e inflação apresentam um impacto significante e negativo sobre o índice de confiança do consumidor britânico. Os determinantes da confiança do consumidor na Bélgica foram investigados por Vuchelen (2004), cuja análise apontou para uma influência negativa da taxa de desemprego e taxa de juros de curto prazo, e uma influência positiva do índice do ciclo comercial e nas expectativas de crescimento. McIntyre (2007) encontrou evidências de que a confiança do consumidor norte-americano é negativamente afetada pela inflação e pela taxa de desemprego, e afetada positivamente pela taxa de juros, mercado de ações e crescimento da renda.

Por fim, quanto à terceira categoria de variáveis, a qual inclui os fatores políticos, acontecimentos internacionais e outros fatores exógenos, Vuchelen (1995) salientou que mudanças de governo ou notícias políticas como eleições podem ter impactos na confiança do consumidor. Ademais, Malgarini e Margani (2007) encontraram evidências de que, além das variáveis convencionais utilizadas para prever os determinantes da confiança do consumidor na Itália, fatores políticos 
(como eleições ou entrada na Zona do Euro) e fatores internacionais (como o 11 de setembro e a guerra do Kosovo) possuem influências significantes sobre a confiança do consumidor. De maneira sucinta, a Tabela 1 apresenta um resumo dos estudos empíricos sobre os determinantes da confiança do consumidor em diversos países.

Tabela 1. Sumário de Estudos Empíricos

\begin{tabular}{|c|c|c|}
\hline Autor (es) & $\begin{array}{l}\text { Local/Período/ } \\
\text { Metodologia }\end{array}$ & Conclusões \\
\hline De Boef e Kellstedt (2004) & $\begin{array}{l}\text { EUA (1981-2002). } \\
\text { Frequência mensal. } \\
\text { VAR NEC }\end{array}$ & $\begin{array}{l}\text { A CC afeta as percepções da economia no } \\
\text { curto e longo prazos. Efeitos políticos } \\
\text { também interferem. }\end{array}$ \\
\hline Golinelli e Parigi (2004) & $\begin{array}{l}8 \text { países (1970-2002). } \\
\text { Frequência trimestral. } \\
\text { VAR }\end{array}$ & $\begin{array}{l}\text { Os ICC têm alguma capacidade de prever a } \\
\text { evolução da atividade econômica. }\end{array}$ \\
\hline Çelik (2010) & $\begin{array}{l}\text { Turquia. } \\
\text { Frequência mensal. } \\
\text { MQO }\end{array}$ & $\begin{array}{l}\text { Movimentos na CC dependem de } \\
\text { mudanças na taxa de câmbio, índice de } \\
\text { bolsa, índice da indústria de manufatura e } \\
\text { expectativa. }\end{array}$ \\
\hline Bachmann e Sims (2012) & $\begin{array}{l}\text { EUA. Frequência } \\
\text { mensal e trimestral. } \\
\text { VAR }\end{array}$ & $\begin{array}{l}\text { Importância da CC como mecanismo de } \\
\text { transmissão de políticas econômicas. }\end{array}$ \\
\hline $\begin{array}{l}\text { Ramalho, Caleiro e } \\
\text { Dionfsio (2011) }\end{array}$ & $\begin{array}{l}\text { Portugal (1987-2009). } \\
\text { Frequência mensal. } \\
\text { VAR }\end{array}$ & $\begin{array}{l}\text { CC essencialmente explicada pelo } \\
\text { desemprenho econômico, entrada na zona } \\
\text { do euro e fatores políticos. }\end{array}$ \\
\hline Karasoy (2015) & $\begin{array}{l}\text { Turquia. Frequência } \\
\text { mensal. MQO/MQG }\end{array}$ & $\begin{array}{l}\text { ICCs afetados pela volatilidade financeira de } \\
\text { forma a deteriorá-los. }\end{array}$ \\
\hline Gürgür e Kilinç (2015) & $\begin{array}{l}\text { Turquia (2005-2015). } \\
\text { Frequência mensal. } \\
\text { ARDL }\end{array}$ & $\begin{array}{l}\text { Principais determinantes da CC: preços ao } \\
\text { consumidor, taxas de câmbio, juros e } \\
\text { desemprego. }\end{array}$ \\
\hline
\end{tabular}

Fonte: Elaboração Própria. Notas: CC - Confiança do Consumidor; ICC - Índice de Confiança do Consumidor.

Assim, observa-se que a confiança do consumidor mantem relações significantes com a economia real, sejam elas macroeconômicas, financeiras ou políticas. Ademais, observa-se que os estudos que analisam os determinantes da confiança do consumidor são em menor quantidade e que, para o Brasil, foram encontrados apenas estudos referentes à capacidade preditiva da confiança do consumidor. Feita a revisão da literatura empírica acerca dos determinantes da confiança do consumidor, parte-se, na próxima seção, para a análise empírica do caso brasileiro, objetivo deste artigo. 


\section{Metodologia Econométrica e Dados 3.1. Metodologia ARDL}

A fim de analisar o que determina a confiança do consumidor e sua dinâmica sobre a política monetária brasileira, propõe-se o uso dos modelos Autorregressivos de Defasagem Distribuída (ARDL) aliados à abordagem de cointegração (Bounds Testing ARDL Approach to Cointegration), com a possibilidade de testar a existência de uma relação entre uma variável dependente e seus regressores, independentemente da ordem de integração destas variáveis. Esta metodologia foi proposta nos trabalhos de Pesaran e Shin (1999) e Pesaran et al. $(1999,2001)$.

Pesaran et al. (2001) argumentaram que os estudos já existentes até os seus escritos concentravam em casos em que as variáveis subjacentes eram integradas de ordem 1, I(1). Isto envolvia, inevitavelmente, um certo grau de "pré-ensaio", introduzindo assim um nível de incerteza para a análise dos níveis de relacionamento. Os autores propuseram uma nova abordagem para testar uma relação entre as variáveis em nível, que é aplicável independentemente se os regressores subjacentes são puramente I(0), puramente I(1) ou mutuamente cointegrados $^{1}$. Os autores propuseram também um nível ótimo de defasagem que pode ser determinado previamente por meio de um critério de seleção.

O modelo ARDL é estimado na forma de um modelo de vetores de correção de erros (ARDL-ECM). Significa que, uma vez que confirmada a existência de uma relação de longo prazo, podem ser estimados os coeficientes de curto e longo prazos, além da velocidade de ajustamento ao equilíbrio de longo prazo. A equação a seguir expressa o modelo ARDL-ECM

$$
\Delta y_{t}=\alpha_{0}+\alpha_{1}\left\ulcorner+\delta_{1} y_{t-1}+\delta_{2} x_{t-1}+\sum_{i=1}^{n} \phi_{1 i} \Delta y_{t-\mathrm{i}}+\sum_{i=0}^{n} \phi_{2 i} \Delta x_{t-\mathrm{i}}+\varepsilon_{t}\right.
$$

em que $\Delta$ representa a primeira diferença; $\alpha_{0}$ e $\alpha_{1}$ são os termos da constante e tendência; $\delta_{1}$ e $\delta_{2}$ são os parâmetros de longo prazo; $\phi_{1}$ e $\phi_{2}$ são os parâmetros de curto prazo; $\boldsymbol{\varepsilon}_{\boldsymbol{t}}$ é o termo de erro.

A estatística subjacente ao teste de cointegração proposto por Pesaran et al. (2001) é a estatística F, responsável por testar a significância conjunta dos parâmetros de longo prazo, um tipo de Dickey-Fuller generalizado a fim de testar

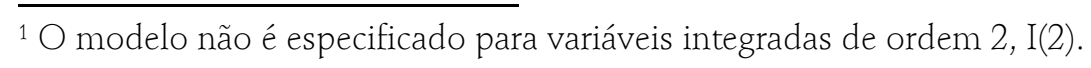


a significância das variáveis em nível em questão, e um equilíbrio condicional do modelo de correção de erros (ECM) já demostrado na equação 1 .

É relevante fazer alguns testes de diagnóstico antes de prosseguir com as análises das estimativas de curto e longo prazos dos modelos ARDL. O Teste LM de Autocorrelação é um tipo destes testes de diagnósticos, cuja hipótese nula é de inexistência de autocorrelação serial nos resíduos. Outro conjunto de testes foi proposto por Brown, Durbin e Evans (1975), no intuito de analisar a estabilidade dos parâmetros das regressões estimadas. Estes dois testes, Soma Cumulativa dos Resíduos Recursivos (CUSUM) e Soma Cumulativa dos Quadrados dos Resíduos Recursivos (CUSUMO), permitem observar a constância dos parâmetros em um modelo. A hipótese nula é a de estabilidade dos coeficientes (Vieira e Da Silva, 2016)

Para qualquer ordem de integração dos regressores ${ }^{2}$, em conformidade com a hipótese nula de não existência de coitegração, Pesaran et al. (2001) propuseram dois conjuntos de valores críticos que fornecem uma banda cobrindo todas as classificações possíveis dos regressores. Isto significa que o limite inferior será calculado sob a hipótese de que todas as variáveis do modelo ARDL são puramente integradas de ordem 0 , isto é, são estacionárias e o limite superior sob a hipótese de que todas as variáveis são puramente integradas de ordem 1, I(1).

Ainda de acordo com Pesaran et al. (2001), uma vez conhecida esta banda de valores críticos, a estatística F é comparada com tais valores da banda. Sendo assim, a hipótese nula do modelo está baseada na não existência de cointegração, $H_{0}: \delta_{1}=\delta_{2}=0$ contra a hipótese alternativa de existência de relação de longo prazo entre as variáveis, $H_{1}: \delta_{1} \neq \delta_{2} \neq 0$. Caso a estatística $F_{\text {calculada }}$ caia fora dos limites de valores críticos, podem ser retiradas inferências conclusivas sem a necessidade de saber a ordem de integração/cointegração dos regressores. Uma vez que caindo abaixo da banda inferior, a hipótese nula não será rejeitada. Ao cair acima da banda superior, a hipótese nula é rejeitada. Contudo, se a estatística $F_{\text {calculada }}$ cair dentro desses limites, a inferência é inconclusiva e o conhecimento do estado de integração das variáveis subjacentes ao modelo é necessário a priori.

${ }^{2}$ Desde que I(0) ou I(1), uma vez que o modelo não é especificado para variáveis I(2). 


\subsection{Descrição das Variáveis Utilizadas}

Para a construção da base de dados propõe-se o uso das variáveis apresentadas na Tabela 2, sintetizando a descrição e fonte dos dados a serem utilizados nas estimações econométricas, referentes ao período de janeiro de 2003 a dezembro de 2016 .

Tabela 2. Variáveis do Modelo

\begin{tabular}{|c|c|c|c|}
\hline Variáveis & Definição & $\begin{array}{l}\text { Unidade de } \\
\text { Medida }\end{array}$ & Fonte \\
\hline LICC & Logaritmo do Índice de Confiança do Consumidor & Índice & OCDE \\
\hline LIPCA & $\begin{array}{l}\text { Logaritmo Índice de Preços ao Consumidor Amplo } \\
\text { Dessazonalizado (Dez/2010 = 100) }\end{array}$ & $\mathrm{N}^{\circ}$ índice & $\mathrm{BCB}$ \\
\hline LLFT & $\begin{array}{l}\text { Logaritmo dos Títulos públicos federais - Letras } \\
\text { Financeiras do Tesouro (fora do BC) - LFT - fim } \\
\text { período }\end{array}$ & $\mathrm{R} \$$ (milhões) & $\mathrm{BCB}$ \\
\hline LIBCBR & $\begin{array}{l}\text { Logaritmo do Índice de Atividade Econômica do } \\
\text { Banco Central do Brasil com Ajuste Sazonal - IBC-Br }\end{array}$ & Índice & $\mathrm{BCB}$ \\
\hline PED & Taxa de Desemprego Dessazonalizada & $\%$ & Seade/PED \\
\hline SALÁRIO & $\begin{array}{l}\text { Logaritmo do rendimento médio real do trabalho } \\
\text { principal, efetivamente recebido no mês de referência, } \\
\text { pelas pessoas de } 10 \text { anos ou mais de idade, ocupadas } \\
\text { na semana de referência, deflacionado e } \\
\text { dessazonalizado. }\end{array}$ & $\mathrm{R} \$$ & IBGE \\
\hline SELIC & Taxa Selic Over Diária & $\%$ a.a. & $\mathrm{BCB}$ \\
\hline LERR & Logaritmo da Taxa de Câmbio Real Efetiva & $\mathrm{n}^{\circ}$ índice & BIS \\
\hline DELEI & Dummy eleição 2014 & - & - \\
\hline
\end{tabular}

Fonte: Elaboração Própria. Notas: BCB (2017), BIS(2017), IBGE(2017), OCDE(2017) e SEADEPED(2017).

A Organização para a Cooperação e Desenvolvimento Econômico (OCDE) é responsável por calcular um indicador de confiança para diversos países do mundo. $\mathrm{O}$ índice se baseia em algumas investigações sobre os planos das famílias para grandes aquisições e opinião sobre a situação econômica do país, sejam elas atuais ou futuras. Optou-se, então, por utilizar o índice calculado pela OCDE neste trabalho pelo fato de, em pesquisas futuras, haver a possibilidade de comparar os índices com outros países, necessitando assim de uma base de dados uniforme ${ }^{3}$.

O comportamento da variável dependente, LICC, durante o período de análise está representado no Gráfico 1. É importante destacar que a confiança do consumidor brasileiro seguiu uma tendência de alta de 2003 até início de 2013,

\footnotetext{
${ }^{3}$ Outras entidades também calculam Índices de Confiança do Consumidor para o Brasil. Por exemplo: Instituto Brasileiro de Economia (IBRE) da Fundação Getúlio Vargas (FGV); Federação do Comércio do Estado de São Paulo (FECOMERCIO-SP); Confederação Nacional da Indústria (CNI).
} 
percebendo uma queda durante o período da crise internacional de 2008. Contudo, nem mesmo este episódio fez com que tal confiança caísse tanto quanto durante o ano de 2013 a 2015, cuja queda pode estar associada ao período das eleições presidenciais e as repercussões desta sobre a confiança. A incerteza quanto à situação econômica do país durante esse período caiu de maneira substancial e isso fez com que as expectativas dos consumidores se deteriorassem, piorando o indicador de confiança. A Tabela 1.A do apêndice estatístico contém as estatísticas descritivas para o período em análise.

Gráfico 1. Índice de Confiança do Consumidor do Brasil - OCDE

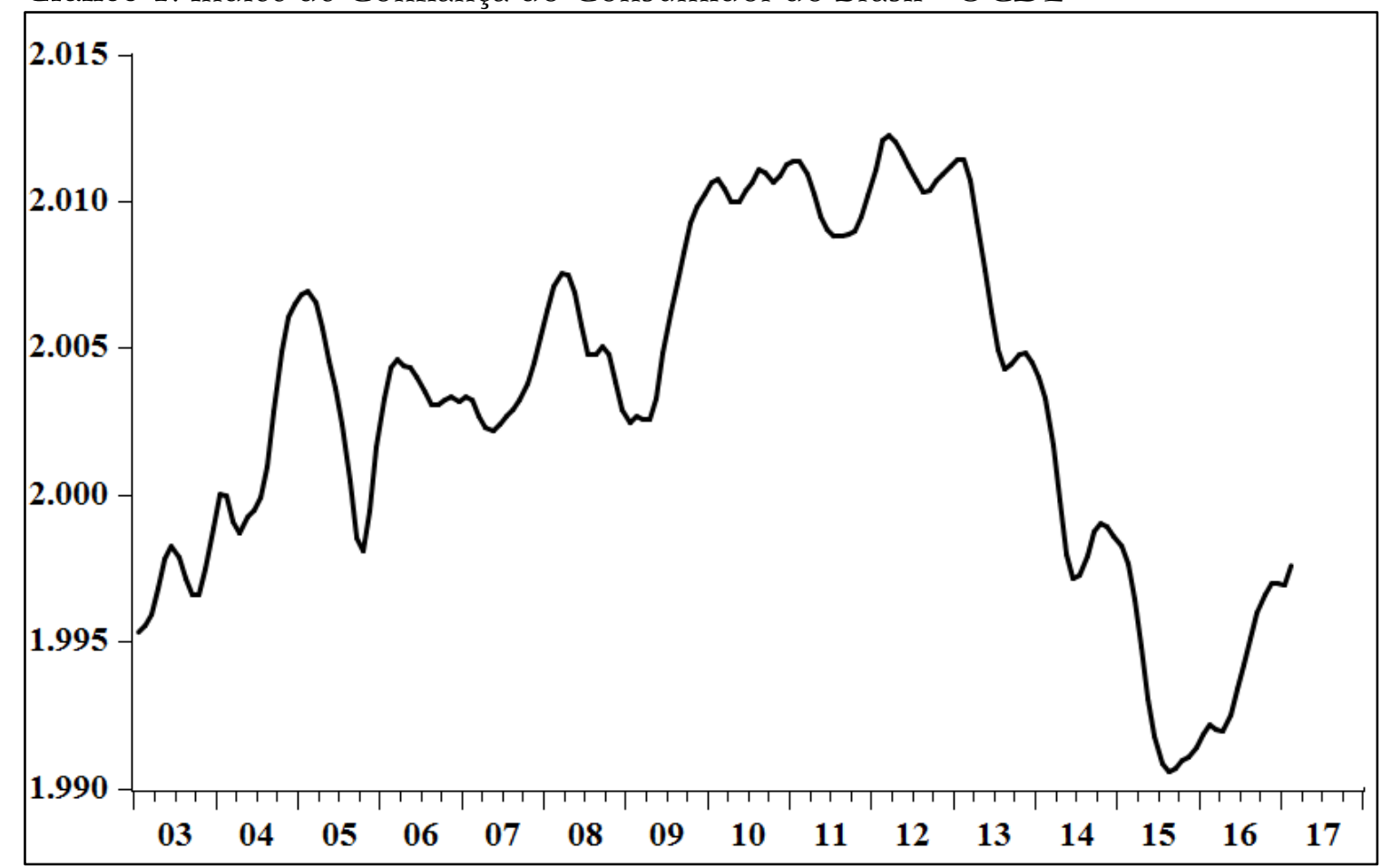

Fonte: OCDE (2017)

\subsection{Estratégia Empírica}

A proposta colocada para este estudo de formalizar a determinação da confiança do consumidor brasileiro num modelo ARDL aliado à cointegração se dá a fim de delinear a elaboração da política monetária no país com vistas a mensurar com mais efetividade o canal das expectativas.

Para tanto, serão estimados três modelos de forma que o primeiro será representado de forma mais geral e o último um modelo mais bem especificado. A escolha por esta forma de apresentação tem por objetivo demonstrar como a inclusão de variáveis que se julga relevantes para determinar o índice de confiança 
do Brasil é realizada e suas implicações para o resultado. Os três modelos estão representados a seguir. Do modelo 1 para o modelo 2 foi acrescentada apenas uma variável categórica, denominada DELEI, para tentar captar os efeitos políticos. Já no modelo 3 , além das variáveis utilizadas nos modelos 1 e 2 , foram acrescentadas outras variáveis de controle (salário e taxa de desemprego).

$$
\begin{gathered}
\text { Modelo } 1: \Delta(\text { LICC })_{t}=\alpha_{0}+\alpha_{1 \Gamma}+\beta_{1}(\text { LICC })_{t-1}+\beta_{2}(\text { SELIC })_{t-1}+\beta_{3}(\text { LERR })_{t-1}+\beta_{4} \\
(\text { LIBCBR })_{t-1}+\beta_{5}(\text { LIPCA })_{t-1}+\sum_{i=0}^{n} \beta_{6} \Delta(\text { LICC })_{t-i}+\sum_{i=0}^{n} \beta_{7} \Delta(\text { SELIC })_{t-i}+ \\
\sum_{i=0}^{n} \beta_{8} \Delta(L E R R)_{t-i}+\sum_{i=0}^{n} \beta_{9} \Delta(\text { LIBCBR })_{t-i}+\sum_{i=0}^{n} \beta_{10} \Delta(\text { LIPCA })_{t-i}+u_{t}
\end{gathered}
$$

$$
\begin{gathered}
\text { Modelo 2: } \Delta(\text { LICC })_{t}=\alpha_{0}+\alpha_{1 \Gamma}+\beta_{1}(\text { LICC })_{t-1}+\beta_{2}(\text { SELIC })_{t-1}+\beta_{3}(L E R R)_{t-1} \\
+\beta_{4}(\text { LIBCBR })_{t-1}+\beta_{5}(L I P C A)_{t-1}+\beta_{6}(D E L E I)_{t-1} \sum_{i=0}^{n} \beta_{7} \Delta(L I C C)_{t-i}+ \\
\sum_{i=0}^{n} \beta_{8} \Delta(\text { SELIC })_{t-i}+\sum_{i=0}^{n} \beta_{9} \Delta(L E R R)_{t-i}+\sum_{i=0}^{n} \beta_{10} \Delta(L I B C B R)_{t-i}+ \\
\sum_{i=0}^{n} \beta_{11} \Delta(\text { LIPCA })_{t-i}+u_{t}
\end{gathered}
$$

$$
\begin{gathered}
\text { Modelo 3: } \Delta(\text { LICC })_{t}=\alpha_{0}+\alpha_{1 \Gamma}+\beta_{1}(\text { LICC })_{t-1}+\beta_{2}(\text { SELIC })_{t-1}+\beta_{3}(\text { LERR })_{t-1}+\beta_{4} \\
\begin{array}{c}
(\text { LIBCBR })_{t-1}+\beta_{5}(\text { LIPCA })_{t-1}+\beta_{6}(\text { DELEI })_{t-1}+\beta_{7}(\text { Salário })_{t-1}+\beta_{8}(P E D)_{t-1}+ \\
\sum_{i=0}^{n} \beta_{9} \Delta(\text { LICC })_{t-i}+\sum_{i=0}^{n} \beta_{10} \Delta(\text { SELIC })_{t-i}+\sum_{i=0}^{n} \beta_{11} \Delta(L E R R)_{t-i}+\sum_{i=0}^{n} \beta_{12} \Delta \\
(\text { LIBCBR })_{t-i}+\sum_{i=0}^{n} \beta_{13} \Delta(\text { LIPCA })_{t-i}+\sum_{i=0}^{n} \beta_{14} \Delta(L L F T)_{t-i}+ \\
\sum_{i=0}^{n} \beta_{15} \Delta(\text { Salário })_{t-i}+\sum_{i=0}^{n} \beta_{16} \Delta(\text { PED })_{t-i}+u_{t}
\end{array}
\end{gathered}
$$

\section{Resultados}

Como já descrito anteriormente, a abordagem ARDL possui a vantagem de aplicação sem antes conhecer a ordem de integração das variáveis. Contudo, caso o teste dos limites seja inconclusivo será necessário conhecer a ordem de integração da variável para que se rejeite a hipótese nula de não cointegração. Sendo assim, na Tabela 3 estão calculados os seguintes testes de raiz unitária: Augmented DickeyFuller (ADF), Phillips-Perron (PP), Kwiatkowski-Phillips-Schmidt-Shin (KPSS) e o Modified Dickey-Fuller Test (DF-GLS). Com exceção do KPSS, todos os testes indicam que as variáveis utilizadas na estimação são integradas de ordem 1, portanto, são não estacionárias ao nível de 1\% de significância estatística. 
Tabela 3. Testes de Raiz Unitária

\begin{tabular}{c|cccc}
\hline \hline Variáveis & ADF & PP & KPSS & DF-GLS \\
\hline LICC & $-1,76$ & $-1,81$ & $0,32^{*}$ & $-1,23$ \\
\hline LIPCA & $-1,07$ & $-0,78$ & $0,32^{*}$ & $-1,37$ \\
\hline LLFT & $-1,63$ & 0,24 & 0,10 & $-2,32$ \\
\hline LIBCBR & $-0,77$ & $-0,33$ & $0,33^{*}$ & $-0,79$ \\
\hline PED & 1,68 & 1,78 & $0,31^{*}$ & 0,34 \\
\hline Salário & $-1,23$ & $-2,37$ & $0,18^{* *}$ & $-1,18$ \\
\hline Selic & $-4,63^{*}$ & $-2,42$ & $0,32^{*}$ & $-1,62$ \\
\hline LERR & $-2,53$ & $-2,08$ & $0,36^{*}$ & $-1,33$ \\
\hline \hline
\end{tabular}

Notas: ADF, PP e DF-GLS: $H_{0}=$ série com raiz unitária. KPSS: $H_{0}=$ série estacionária; ${ }^{*} e^{* *}$ denotam rejeição da hipótese nula a 1\% e 5\% de nível de significância, respectivamente.

A Tabela 4 reporta as estimativas dos modelos ARDL com um máximo de quatro defasagens para cada parâmetro, sendo selecionados pelo Critério de Akaike (AIC). A ordem das variáveis segue os modelos 1, 2 e 3 já supracitados. Além das estimativas, estão reportados os testes LM de autocorrelação, cuja hipótese nula é a de que há ausência de autocorrelação. Para os três modelos percebe-se que o componente defasado da confiança do consumidor desempenha um papel importante. Além disso, os valores passados de outras duas variáveis possuem poder explicativo na confiança do consumidor, quais sejam, índice de preços ao consumidor e salário.

Tabela 4. Estimativas dos Modelos ARDL (Variável Dependente: Índice de Confiança do Consumidor

\begin{tabular}{|c|c|c|c|}
\hline Modelo & $\begin{array}{l}\text { Defasagens } \\
\text { Selecionadas }\end{array}$ & $\begin{array}{c}\text { ARDL } \\
\text { Variáveis Significantes } \\
\text { (Defasagens significantes entre parêntesis) }\end{array}$ & $\begin{array}{c}\text { Teste LM } \\
\text { Autocorrelação } \\
{[\text { Prob] }}\end{array}$ \\
\hline 1 & $(4,0,0,0,2)$ & $\begin{array}{c}\text { Índice de Confiança }(-1,-2,-3,-4) ; \text { Selic (0); LERR } \\
(0) ; \text { LIBCBR }(0) \text {; LIPCA }(-1,-2)\end{array}$ & $\begin{array}{c}2,713 \\
{[0,257]}\end{array}$ \\
\hline 2 & $(4,0,0,0,2,0)$ & $\begin{array}{l}\text { Índice de Confiança }(-1,-2,-3,-4) ; \text { Selic (0); LERR } \\
(0) ; \operatorname{LIBCBR}\left(0^{*}\right) ; \operatorname{LIPCA}(-1,-2)\end{array}$ & $\begin{array}{l}3.128 \\
{[0,209]}\end{array}$ \\
\hline 3 & $(4,0,1,0,1,0,4$ & $\begin{array}{l}\text { Índice de Confiança (-1, }-2,-3,-4) \text {; Selic (0); LERR } \\
(-1) \text {; LIBCBR (0); LIPCA (-1); LFT (0); Salário }\left(0^{*},-\right. \\
4)\end{array}$ & $\begin{array}{c}0,555 \\
{[0,757]}\end{array}$ \\
\hline
\end{tabular}

Fonte: Elaboração própria. Notas: Modelo ARDL com um máximo de 04 defasagens para cada modelo. Modelo 2 inclui uma dummy. Escolha do modelo baseada no Critério de Informação de Akaike. Asterisco (*) indica significância somente a 10\%. Todas as variáveis estão em log natural, com exceção da Selic e PED. 
Para que as dinâmicas de curto e longo prazos sejam analisadas é necessário realizar alguns testes de diagnóstico para avaliar se as análises futuras são confiáveis. No que se refere à autocorrelação, para os três modelos, não se rejeita a hipótese nula, inferindo que os modelos não sofrem deste problema. Estes resultados podem ser vistos na última coluna da Tabela 4. Quanto aos testes de estabilidade CUSUM e CUSUMSQ, apresentados nas Figuras 1, 2 e 3, pode-se dizer que os parâmetros dos três modelos são estáveis, com exceção apenas do CUSUM do modelo 2 , que apresentou uma instabilidade bem pequena.

Figura 1. Testes de Estabilidade CUSUM e CUSUMSO do Modelo 1
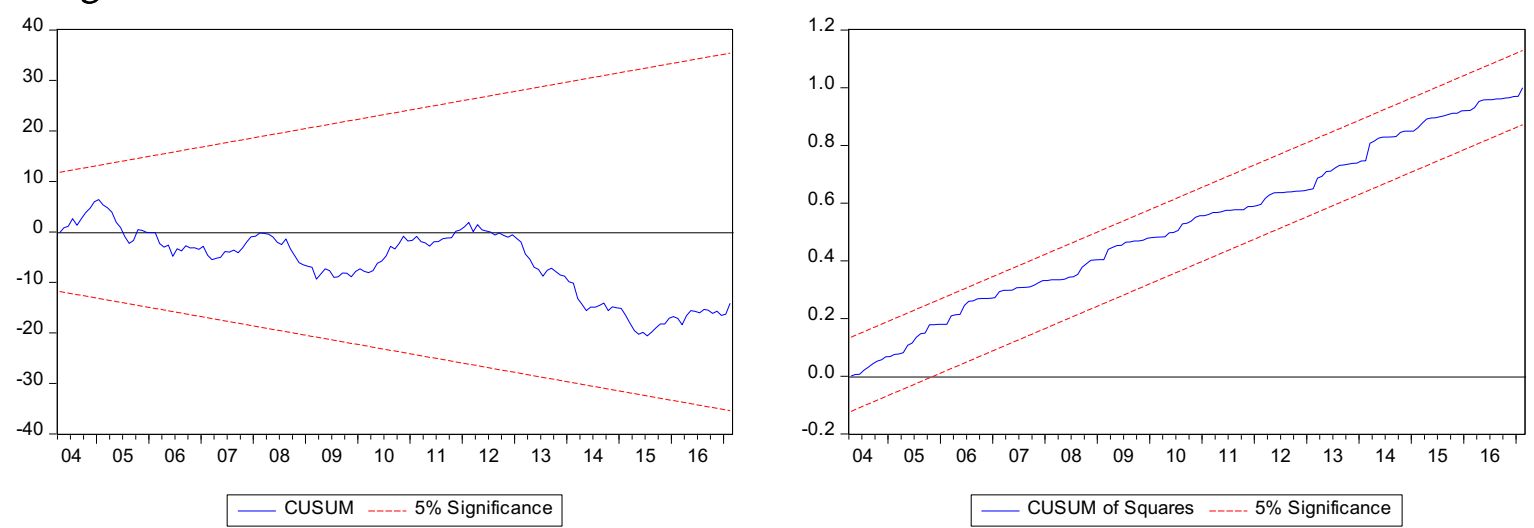

Fonte: Elaboração Própria

Figura 2. Testes de Estabilidade CUSUM e CUSUMSQ do Modelo 2
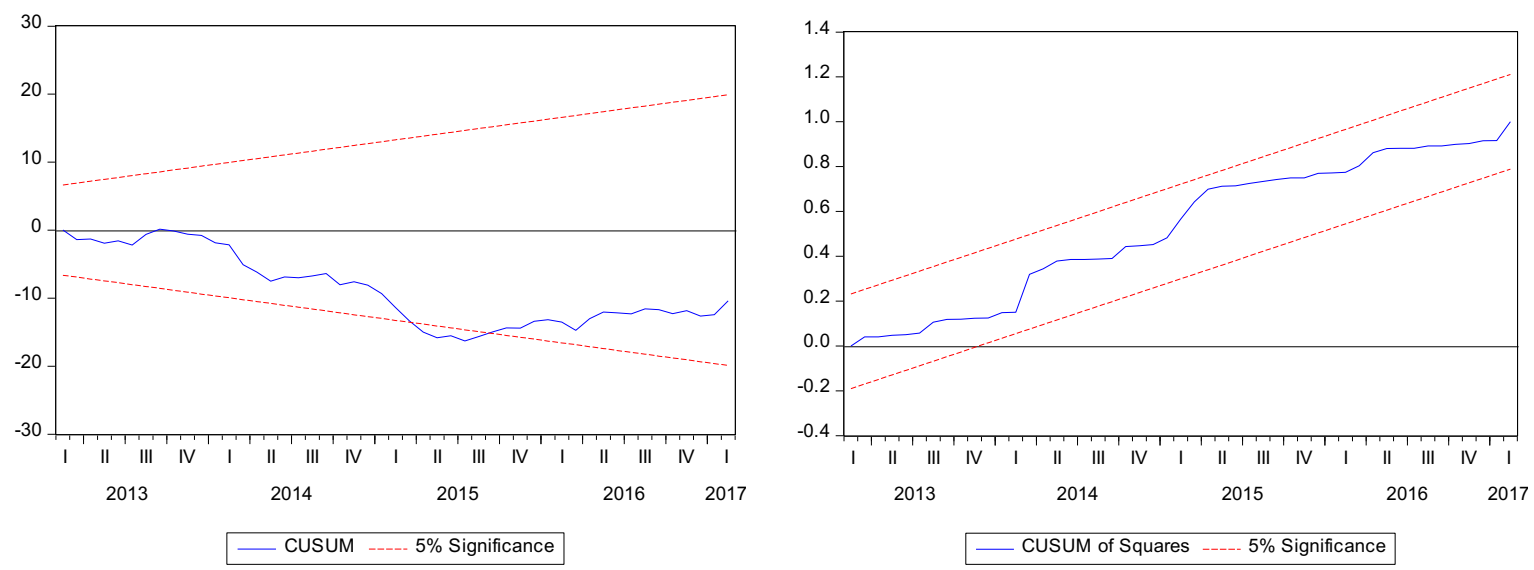

Fonte: Elaboração Própria 
Figura 3. Testes de Estabilidade CUSUM e CUSUMSO do Modelo 3
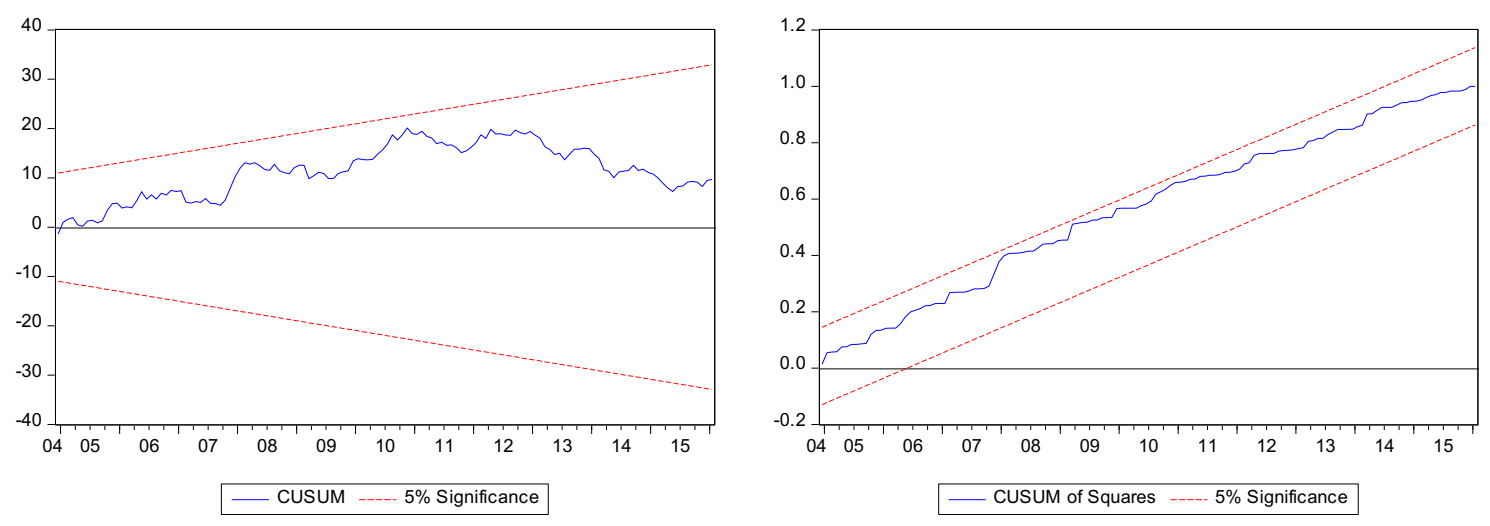

Fonte: Elaboração Própria

Para analisar a existência de vetores e cointegração entre as variáveis, aplicou-se a abordagem de Testes de Fronteira (Bounds Testing) de Pesaran et al. (2001), descritos na Tabela 5. A hipótese nula de inexistência de relação de longo prazo pode ser rejeitada a $5 \%$ de significância para o modelo 3 , pois a estatística $\mathrm{F}$ é maior que os limites propostos por Pesaran et al. (2001). Já para os modelos $1 \mathrm{e}$ 2 rejeita-se a hipótese nula a $10 \%$ e é inconclusivo a $5 \%$. Entretanto, os testes de raiz unitária expostos na Tabela 3 ajudam a concluir que as variáveis podem ser cointegradas ${ }^{1}$.

Tabela 5. Teste de Cointegração ARDL - Teste dos Limites (Bounds)

\begin{tabular}{|c|c|c|c|c|c|c|}
\hline \multirow{3}{*}{ Modelo } & \multirow{3}{*}{ Estatística F } & \multicolumn{4}{|c|}{ Valores Críticos } & \multirow{3}{*}{$\begin{array}{c}\text { Há } \\
\text { Cointegração? }\end{array}$} \\
\hline & & \multicolumn{2}{|c|}{ Limite $I(0)$} & \multicolumn{2}{|c|}{ Limtie I(1) } & \\
\hline & & $10 \%$ & $5 \%$ & $10 \%$ & $5 \%$ & \\
\hline 1 & 3,42 & 2,20 & 2,56 & 3,09 & 3,49 & $\begin{array}{c}\text { Sim a } 10 \% \\
\text { inconclusivo a } 5 \%\end{array}$ \\
\hline 2 & 3,09 & 2,08 & 2,39 & 3,00 & 3,38 & $\begin{array}{c}\text { Sim a } 10 \%, \\
\text { inconclusivo a } 5 \%\end{array}$ \\
\hline 3 & 5,88 & 2,22 & 2,50 & 3,17 & 3,50 & Sim \\
\hline
\end{tabular}

Nota: $\mathrm{H}_{0}$ (não há relação de longo prazo).

Este resultado encontrado é, por si só, muito interessante na análise dos determinantes da confiança do consumidor no caso brasileiro. Ele aponta que as

\footnotetext{
${ }^{1}$ Conhecer a ordem de integração das variáveis auxilia em escolher um método (tipo Johansen ou EG que exigem que as variáveis sejam I(1)).
} 
variáveis macroeconômicas analisadas têm papel importante de longo prazo nas tomadas de decisão dos agentes econômicos. Mas é importante uma investigação do papel de cada variável estimada neste contexto de longo prazo. Além disso, esta relação de longo prazo não significa que o sistema está isento de choques de curto prazo, o que também deve ser investigado. Esses serão os próximos passos.

Após verificar a existência de vetores de cointegração, foram estimados os coeficientes de longo prazo apresentados na Tabela 6. É importante ressaltar alguns pontos para os três modelos estimados e, além disso, analisar as diferenças quando variáveis são incluídas nas estimações. No modelo mais geral (modelo 1), o índice de confiança do consumidor brasileiro depende principalmente da taxa de juros Selic e da taxa de câmbio, assim como no modelo 2 . A relação negativa entre a taxa Selic e o índice de confiança do consumidor indica que uma taxa de juros maior faz com que os consumidores se tornem mais cautelosos, reduzindo suas expectativas quanto à situação econômica do país, o que é de se esperar. Vale salientar que tanto em termos de sinal, quanto de magnitude, os coeficientes de longo prazo para a taxa de juros se mantiveram para os três modelos. Assim, verifica-se que o instrumento de política monetária possui uma relação estável de longo prazo com a confiança do consumidor. Desta forma, as decisões do Banco Central de elevar a taxa de juros, medido pela taxa Selic, em um ponto percentual irá reduzir, a longo prazo, a confiança dos consumidores brasileiros em 0,15 \%. A taxa de câmbio efetiva real, por sua vez, apresentou uma relação positiva com o índice de confiança do consumidor para os modelos 1 e 2 (neste último ela é significante apenas ao nível de 10\%).

Como já descrito, a inclusão de variáveis que mensuram efeitos políticos ou eventos internacionais são comuns para investigar os determinantes da confiança do consumidor. Neste sentido, incluiu-se uma dummy para as eleições de 2014 no modelo 2, período pelo qual o índice de confiança dos consumidores brasileiros apresentou uma forte queda, como foi observado no Gráfico 1. Embora a dummy incluída no modelo tenha apresentado uma relação negativa com o índice, demonstrando que os acontecimentos políticos do período considerado deterioraram a confiança, tal coeficiente não apresentou resultados estatisticamente significantes. 
Tabela 6. Modelos ARDL: Coeficientes de Longo Prazo (Variável Dependente: Índice de Confiança do Consumidor)

\begin{tabular}{cccc}
\hline Modelo & 1 & 2 & 3 \\
\hline (Modelo ARDL $)$ & $(4,0,0,0,2)$ & $(4,0,0,0,2,0)$ & $(4,0,1,0,1,0,4,0)$ \\
\hline \multirow{2}{*}{ Variáveis } & Coefic. & Coefic. & Coefic. \\
& {$[$ Prob. $]$} & {$[$ Prob. $]$} & {$[$ Prob. $]$} \\
\hline \multirow{2}{*}{ Selic } & $-0,002$ & $-0,002$ & $-0,0015$ \\
& {$[0,055]$} & {$[0,051]$} & {$[0,000]$} \\
LERR & 0,093 & 0,076 & $-0,017$ \\
& {$[0,040]$} & {$[0,071]$} & {$[0,116]$} \\
LIBCBR & $-0,323$ & $-0,252$ & 0,189 \\
& {$[0,159]$} & {$[0,219]$} & {$[0,000]$} \\
LIPCA & 0,028 & 0,0145 & 0,608 \\
& {$[0,600]$} & {$[0,763]$} & {$[0,000]$} \\
LLFT & - & - & 0,069 \\
& & - & {$[0,000]$} \\
Salário & - & - & 0,400 \\
& & - & $-0,000]$ \\
PED & - & {$[0,392]$} & {$[0,819]$} \\
& & & - \\
Dummy & - & & \\
\end{tabular}

Notas: Todas as variáveis estão em log natural, com exceção da Selic e PED. Coeficientes marcados em negrito possuem significância estatística de até $10 \%$.

A inclusão das variáveis taxa de desemprego (PED) e das letras financeiras do tesouro (LLFT) no modelo 3 fez com que as estimativas do IBC-Br, proxy do PIB (LIBCBR), e índice de preços ao consumidor (LIPCA) dos modelos 1 e 2 se tornassem estatisticamente significantes. Sendo assim, no modelo 3 o índice de confiança do consumidor brasileiro depende principalmente do índice de preços ao consumidor, do salário, da proxy do PIB, da LFT e da taxa Selic. É importante salientar que a taxa de inflação (IPCA) apresentou resultado contrário ao esperado ${ }^{2}$ para o período analisado, dado que uma elevação de preços tende a fazer com que os consumidores desistam das grandes aquisições e reduzam suas expectativas quanto ao estado da economia. A relação positiva e estatisticamente significante de longo prazo entre inflação e a confiança do consumidor brasileiro pode levar a uma

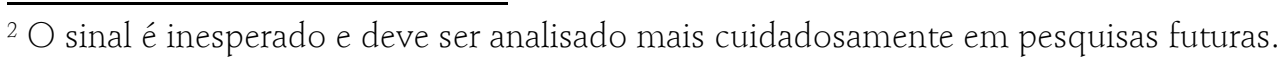


explicação: como as decisões de política monetária são fundamentalmente baseadas no comportamento do consumidor apoiadas na dinâmica de um índice de preços, pode-se dizer que, para o período analisado, o comportamento da demanda foi importante na medida em que elevou os níveis de confiança dos consumidores, dada a alta magnitude de seu coeficiente.

Quanto à proxy do PIB e o salário, a relação positiva do índice do consumidor brasileiro com ambas variáveis é condizente com a teoria, visto que aumentos do salário real facilita a aquisição de produtos e melhora a percepção dos consumidores quanto à situação econômica do país. Este resultado confirma, mais uma vez, a importância do comportamento da demanda na dinâmica econômica, bem como sugere que os consumidores respondem bem aos canais pelos quais a política monetária irá transmitir seus efeitos pretendidos. Da mesma forma, uma elevação do PIB também fará com que as expectativas dos consumidores melhorem. A variável LLFT, que representa a emissão de títulos públicos brasileiros, também apresentou relação positiva com a confiança do consumidor, na medida em que um aumento da remuneração deste título proporciona uma maior confiança para aqueles consumidores que investem neste tipo de mercado. Portanto, estas variáveis (LIBCBR, SALÁRIO e LLFT) criam surtos otimistas nos consumidores, melhorando assim a sua confiança e fornecendo evidências a favor de um "animal spirits" no Brasil.

É importante ressaltar que foi testado, também, neste modelo mais especificado (modelo 3), os efeitos dos acontecimentos políticos captados pela dummy, DELEI, relativo a forte queda da confiança em 2014. Porém, os resultados não se mostraram estatisticamente significantes nem para o curto e nem para o longo prazo. Portanto, optou-se por não reportar tais estimativas.

Como enfatizado anteriormente, a relação de longo prazo detectada nas estimações não isenta o sistema estimado de receber choques de curto prazo. No entanto, para que a relação de cointegração entre as variáveis seja mantida, há que se ter um mecanismo de correção destes choques para se retornar à dinâmica de longo prazo, caso haja desequilíbrios. Esta é justamente a função do Mecanismo de Correção de Erros.

Assim, seguindo com a análise empírica, o próximo passo é a estimação dos três modelos na forma vetores de correção de erros (ECM) dos modelos ARDL para obter o ajuste de curto prazo reportados na Tabela 7. Para os modelos 1 e 2, os desvios da trajetória de longo prazo da confiança do consumidor são corrigidos, em 
parte, pela variação do próprio índice de confiança defasado, pela taxa de câmbio e o índice de preços ao consumidor com uma defasagem. Como esperado, o termo de correção de erros é negativo e estaticamente significante para os três modelos.

Nos modelos 1 e 2, aproximadamente $2 \%$ do choque é corrigido após o primeiro mês, sugerindo uma velocidade de ajuste bastante baixa. O ECM-ARDL do modelo 3, por sua vez, apresenta uma correção de choque de aproximadamente $9 \%$ para o mês seguinte, também demostrando uma velocidade baixa de ajuste em relação ao equilíbrio de longo prazo. Além disso, os desvios de longo prazo da confiança do consumidor são corrigidos, em parte, pelo próprio índice defasado, taxa de juros Selic, índice de preços ao consumidor, título público e o salário defasado. Estes resultados sugerem que, embora haja uma relação de longo prazo, é possível que haja desvios de curto prazo, e que a correção em direção ao longo prazo é lenta, não chegando a $10 \%$ ao mês. Em outras palavras, variáveis econômicas influenciam a confiança do consumidor e, quando esta confiança é posta em xeque, ela se recupera de forma demorada. Devido a esta lentidão no ajuste, as expectativas dos consumidores possuem recuperação também demorada. Apenas como exemplificação, pois a análise não pode ser feita de forma linear, se há algum desvio na trajetória de longo prazo, e a correção se dá a uma taxa de aproximadamente $10 \%$ ao mês, significa que somente num prazo de $48,31^{3}$ meses é que todo esse choque inicial terá se dissipado.

Tabela 7. Dinâmica de Curto Prazo dos Modelos ARDL: Correção de Erros e Variáveis Significantes

\begin{tabular}{c|c|c}
\hline \hline Modelo & $\begin{array}{c}\text { ECM(-1) } \\
{[\text { Prob. }]}\end{array}$ & $\begin{array}{c}\text { Variáveis Significantes } \\
\text { (Curto Prazo) }\end{array}$ \\
\hline \multirow{2}{*}{1} & -0.019 & Índice de Confiança $(-1,-2,-3) ;$ LERR $(0)$; LIPCA (-1) \\
& {$[0,00]$} & Índice de Confiança $(-1,-2,-3) ;$ LERR (0); LIPCA (-1) \\
\hline \multirow{2}{*}{3} & -0.020 & Índice de Confiança $(-1,-2,-3) ;$ Selic (0); LIPCA ( $\left.0^{*}\right)$ \\
& {$[0,00]$} & LFT (0); Salário $\left(0^{*},-1,-2,-3\right)$ \\
\hline \hline
\end{tabular}

Nota: Todas as variáveis estão em log natural, com exceção da Selic e PED.

Diferentemente das estimativas de longo prazo, o coeficiente de curto prazo do modelo 3 , apresentado na Tabela 8 , demonstrou uma relação negativa entre 0 índice de confiança do consumidor e o índice de preços ao consumidor como era

\footnotetext{
${ }^{3}$ Se o coeficiente do ECM é - 0,10 ou $10 \%$, a meia vida do período de correção é 41,07 meses $(\ln (50)$ $=\mathrm{n} \ln (1+\mathrm{ecm}))$ e o período total é 48,31 meses.
} 
de se esperar. Isto indica que um aumento da inflação causa deterioração da confiança, indicando que as elevações de preços só reduzem a confiança do consumidor no curto prazo.

Tabela 8. Dinâmica de Curto Prazo dos Modelos ARDL

\begin{tabular}{|c|c|c|c|}
\hline & $\begin{array}{c}\text { Modelo } 1 \\
\text { ARDL }(4,0,0,0,2)\end{array}$ & $\begin{array}{c}\text { Modelo } 2 \\
\text { ARDL }(4,0,0,0,2,0)\end{array}$ & $\begin{array}{c}\text { Modelo } 3 \\
\operatorname{ARDL}(4,0,1,0,1,0,4,0)\end{array}$ \\
\hline Regressores & $\begin{array}{l}\text { Coeficiente } \\
\text { [Prob.] }\end{array}$ & $\begin{array}{l}\text { Coeficiente } \\
\text { [Prob.] }\end{array}$ & $\begin{array}{l}\text { Coeficiente } \\
\text { [Prob.] }\end{array}$ \\
\hline$\Delta I C C_{t-1}$ & $\begin{array}{c}1,471 \\
{[0,000]}\end{array}$ & $\begin{array}{c}1,465 \\
{[0,000]}\end{array}$ & $\begin{array}{c}1,361 \\
{[0,000]}\end{array}$ \\
\hline$\Delta I C C_{t-2}$ & $\begin{array}{c}-0,988 \\
{[0,000]}\end{array}$ & $\begin{array}{l}-0,981 \\
{[0,000]}\end{array}$ & $\begin{array}{c}-0,864 \\
{[0,000]}\end{array}$ \\
\hline$\Delta I C C_{t-3}$ & $\begin{array}{c}0,262 \\
{[0,000]}\end{array}$ & $\begin{array}{c}0,257 \\
{[0,000]}\end{array}$ & $\begin{array}{c}0,296 \\
{[0,000]}\end{array}$ \\
\hline$\Delta$ Selic & $\begin{array}{l}-0,000 \\
{[0,132]}\end{array}$ & $\begin{array}{l}-0,000 \\
{[0,146]}\end{array}$ & $\begin{array}{l}-0,000 \\
{[0,016]}\end{array}$ \\
\hline$\triangle L E R R$ & $\begin{array}{c}0,003 \\
{[0,043]}\end{array}$ & $\begin{array}{c}0,003 \\
{[0,040]}\end{array}$ & $\begin{array}{c}0,002 \\
{[0,213]}\end{array}$ \\
\hline$\triangle L I B C B R$ & $\begin{array}{l}-0,004 \\
{[0,537]}\end{array}$ & $\begin{array}{l}-0,003 \\
{[0,667]}\end{array}$ & $\begin{array}{c}0,011 \\
{[0,113]}\end{array}$ \\
\hline$\triangle L I P C A_{t}$ & $\begin{array}{l}-0,035 \\
{[0,202]}\end{array}$ & $\begin{array}{l}-0,042 \\
{[0,131]}\end{array}$ & $\begin{array}{c}-0,044 \\
{[0,100]}\end{array}$ \\
\hline$\triangle L I P C A_{t-1}$ & $\begin{array}{c}0,071 \\
{[0,010]}\end{array}$ & $\begin{array}{c}0,067 \\
{[0,014]}\end{array}$ & X \\
\hline$\triangle D E L E I C$ & - & $\begin{array}{c}-0,000 \\
{[0,685]}\end{array}$ & - \\
\hline$\Delta L L F T$ & - & - & $\begin{array}{c}0,004 \\
{[0,010]}\end{array}$ \\
\hline$\Delta$ Salário $_{t}$ & - & - & $\begin{array}{c}0,007 \\
{[0,068]}\end{array}$ \\
\hline$\Delta$ Salário $_{t-1}$ & - & - & $\begin{array}{l}-0,023 \\
{[0,000]}\end{array}$ \\
\hline$\Delta$ Salário $_{t-2}$ & - & - & $\begin{array}{l}-0,016 \\
{[0,000]}\end{array}$ \\
\hline$\Delta$ Salário $_{t-3}$ & - & - & $\begin{array}{l}-0,010 \\
{[0,015]}\end{array}$ \\
\hline$\triangle P E D$ & - & - & $\begin{array}{c}-0,000 \\
{[0,575]}\end{array}$ \\
\hline
\end{tabular}

Nota: Todas as variáveis estão em log natural, com exceção da Selic e PED; "X" indica que não apresentou valores para esta defasagem; "“" indica que a variável em questão não foi incluída no modelo; Coeficientes marcados em negrito possuem significância de até $10 \%$. 
Já em relação à taxa de juros Selic, verificou-se que tanto a curto como a longo prazo esta taxa apresentou relação negativa com o índice de confiança do consumidor para os três modelos, embora a magnitude destes coeficientes seja pequena.

Uma vez que as decisões de política monetária se baseiam na confiança do consumidor e esta, por sua vez, possui relação estatisticamente significante com o instrumento de política monetária, medida pela Selic, o Banco Central do Brasil deve levar em conta essas expectativas dos consumidores para a tomada de decisões. Entre janeiro de 2003 e dezembro de 2016, portanto, aumentos na taxa de juros Selic deterioraram a confiança do consumidor brasileiro, tanto no curto como no longo prazo. Dessa maneira, estes resultados devem ser levados em consideração pela autoridade monetária na determinação do seu instrumento de política monetária, representada pela taxa de juros Selic neste estudo.

\section{Considerações Finais}

A investigação deste trabalho se baseou nos determinantes da confiança do consumidor brasileiro e a dinâmica de política monetária de janeiro de 2003 a dezembro de 2016, utilizando a metodologia ARDL aliada à cointegração. Os resultados indicaram uma relação de longo prazo entre o índice de confiança do consumidor e demais variáveis macroeconômicas analisadas. Os resultados também indicaram que, a longo prazo, o índice de confiança do consumidor depende do índice de preços do consumidor, do salário real, da taxa de juros Selic, do índice de atividade econômica do Banco Central do Brasil e de um índice de títulos públicos.

Em termos de dinâmica de política monetária, verificou-se a importância do comportamento da demanda, uma vez que a dinâmica de preços ao consumidor se mostrou estatisticamente significante, bem como a relação entre a taxa de juros Selic e a confiança do consumidor brasileiro. Além disso, foram encontradas evidências de que as variáveis econômicas influenciam a confiança do consumidor e, quando esta confiança é posta em xeque, ela se recupera de forma demorada. A variável dummy incluída no modelo, para tentar captar os efeitos políticos, não apresentou resultados significantes para o período de análise.

Desta maneira, a análise aqui proposta demonstra que o canal das expectativas possui relacionamento com as variáveis macroeconômicas. Ademais, em termos de política monetária, o relacionamento estável de longo prazo com o 
instrumento de política, medido pela taxa de juros Selic, indica que a autoridade monetária deve levar em conta estes resultados para a tomada de decisões.

\section{Referências}

Acemoglu, D., \& Scott, A. (1994). Consumer confidence and rational-expectations - are agents beliefs consistent with the theory. Economic Journal, 104(422), 1-19. doi:10.2307/2234671

Akerlof, G. A., \& Shiller, R. J. (2010). Animal spirits: How human psychology drives the economy, and why it matters for global capitalism. Princeton University Press.

Bachmann, R., \& Sims, E. R. (2012). Confidence and transmission of government spending shocks. Journal of Monetary Economics, 59(3), 235-249. doi:10.1016/j.jmoneco.2012.02.005.

Banco Central do Brasil. (2011). Dez anos de metas para a inflação - 1999-2009. Brasília: Banco Central do Brasil, 456 p.

BCB (2017). Banco Central do Brasil. Disponível em: <https://www3.bcb.gov.br/sgspub/localizarseries/localizarSeries.do?met hod=prepararTelaLocalizarSeries $>$ Acesso em: Mar. 2017

BIS (2017). Bank for Internacional Settlements. Disponível em: <https://www.bis.org/statistics/eer.htm> Acesso em: Mar. 2017.

Belessiotis, T. (1996). Consumer confidence and consumer spending in France. European Commission - Directorate General for Economic and Financial Affairs. Economic Papers, n. 116.

Brown, R. L., Durbin, J., \& Evans, J. M. (1975). Techniques for testing constancy of regression relationships over time. Journal of the Royal Statistical Society Series B-Methodological, 37(2), 149-163.

Carroll, C. D. (1997). Buffer-stock saving and the life cycle/permanent income hypothesis. Quarterly Journal of Economics, 112(1), 1-55. doi:10.1162/003355397555109

Carroll, C. D., Fuhrer, J. C., \& Wilcox, D. W. (1994). Does consumer sentiment forecast household spending - if so, why? American Economic Review, 84(5), 1397-1408. 
Çelik, S., e Özerkek, Y. (2009). Panel cointegration analysis of consumer confidence and personal consumption in the European union. Journal of Business Economics and Management, 10(2), 161-168. doi:10.3846/16111699.2009.10.161-168

Çelik, S. (2010). An unconventional analysis of consumer confidence index for the Turkish Economy. International Journal of Economics and Finance Studies, 2(1), 121-129.

De Boef, S., \& Kellstedt, P. M. (2004). The political (and economic) origins of consumer confidence. American Journal of Political Science, 48(4), 633649. doi:10.1111/j.0092-5853.2004.00092.x

De Mello, E. P. G., \& Figueiredo, F. M. R. (2014). Assessing the short-term forecasting of confidence indices. (Banco Central do Brasil Working Papers, No. 371). Brasilia.

Debes, S., Gareis, J., Mayer, E., Rüth, S. (2014). Towards a consumer channel of monetary policy. Wüzburg Economic Papers, n. 91.

Dees, S., \& Soares Brinca, P. (2013). Consumer confidence as a predictor of consumption spending: Evidence for the United States and the Euro Area. International Economics, 134, 1-14. doi: 10.1016/j.inteco.2013.05.001

Delorme, C. D., Kamerschen, D. R., \& Voeks, L. F. (2001). Consumer confidence and rational expectations in the United States compared with the United Kingdom. Applied Economics, 33(7), 863-869. doi:10.1080/00036840152022205

Dickey, D. A., \& Fuller, W. A. (1979). Distribution of the estimators for autoregressive time-series with a unit root. Journal of the American Statistical Association, 74(366), 427-431. doi:10.2307/2286348

Fan, C. Z. S., \& Wong, P. B. (1998). Does consumer sentiment forecast household spending? The Hong Kong case. Economics Letters, 58(1), 77-84. doi:10.1016/s0165-1765(97)00247-4

SEADE-PED (2017). Fundação Sistema Estadual de Análise de Dados - Pesquisa de Emprego e Desemprego. Disponível em: $<$ http://www.seade.gov.br/produtos/ped-rmsp> Acesso em: Mar. 2017. 
Golinelli, R., \& Parigi, G. (2004). Consumer sentiment and economic activity: a cross country comparison. Journal of Business Cycle Measurement and analysis, 2004(2), 147-170.

Gurgür, T., \& Kilinç, Z. (2015). In search of the Turkish consumer confidence. (Central Bank of the Republic of Turkey, Working Paper, No. 15/38).

IBGE (2017). Instituto Brasileiro de Geografia e Estatística. Disponível em: $<$ https://ww2.ibge.gov.br/home/estatistica/indicadores/trabalhoerendime nto/pme_nova/defaulttab2.shtm > Acesso em: Mar. 2017.

Karasoy, H. G. (2015). Consumer confidence indices and Financial Volatility. (Research and Monetary Policy Department, Central Bank of the Republic of Turkey, No. 2015-16/08).

Katona, G. (1968). Consumer behavior - theory and findings on expectations and aspirations. American Economic Review, 58(2), 19-30.

Keynes, J. M. (1936). The general theory of employment, interest and money. Cambridge University Press.

Kwan, A. C., \& Cotsomitis, J. A. (2006). The usefulness of consumer confidence in forecasting household spending in Canada: A national and regional analysis". Economic Inquiry, 44(1), 185-197. doi:10.1093/ei/cbi064

Kwiatkowski, D., Phillips, P. C. B., Schmidt, P., \& Shin, Y. C. (1992). Testing the null hypothesis of stationarity against the alternative of a unit-root - how sure are we that economic time-series have a unit-root. Journal of Econometrics, 54(1-3), 159-178. doi:10.1016/0304-4076(92)90104-y

Lapp, J. S., \& Pearce, D. K. (2012). The impact of economic news on expected changes in monetary policy. Journal of Macroeconomics, 34(2), 362-379. doi:10.1016/j.jmacro.2012.01.009

Lopes, T. H. C. R., \& De Jesus, C. S. (2016). Consumer confidence and household spending: evidence from Brazil. The Empirical Economics Letters, n. 15/12, 1227-1234.

Ludvigson, S. C. (2004). Consumer confidence and consumer spending. Journal of Economic Perspectives, 18(2), 29-50. doi:10.1257/0895330041371222 
Malgarini, M., \& Margani, P. (2007). Psychology, consumer sentiment and household expenditures. Applied Economics, 39(13-15), 1719-1729. doi:10.1080/00036840600606351

McIntyre, K. H. (2007). Reconciling consumer confidence and permanent income consumption. Eastern Economic Journal, 33(2), 257-275. doi:10.1057/eej.2007.20

OCDE (2017). Organização para Cooperação e Desenvolvimento Econômico. Disponível em: <https://data.oecd.org/leadind/consumer-confidenceindex-cci.htm> Acesso em: Mar. 2017.

Paradiso, A., Kumar, S., \& Margani, P. (2014). Are Italian consumer confidence adjustments asymmetric? A macroeconomic and psychological motives approach. Journal of Economic Psychology, 43, 48-63. doi:10.1016/j.joep.2014.04.006

Pesaran, M. H., \& Shin, Y. (1999). An autoregressive distributed-lag modelling approach to cointegration analysis. Econometrics Society Monographs, n. 31, 371-413.

Pesaran, M. H., Shin, Y., \& Smith, R. J. (1999). Bounds testing approaches to the analysis of long-run relationships. Journal of Statistical Association, n. 94, 621-634.

Pesaran, M. H., Shin, Y., \& Smith, R. J. (2001). Bounds testing approaches to the analysis of level relationships. Journal of Applied Econometrics, 16(3), 289326. doi:10.1002/jae.616

Phillips, P. C. B., \& Perron, P. (1988). Testing for a unit-root in time-series regression. Biometrika, 75(2), 335-346. doi:10.1093/biomet/75.2.335

Pigou, A. C. (1929). Industrial fluctuations. 2nd ed. London: Macmillan.

Ramalho, E. A., Caleiro, A., \& Dionfsio, A. (2011). Explaining consumer confidence in Portugal. Journal of Economic Psychology, 32(1), 25-32. doi:https://doi.org/10.1016/j.joep.2010.10.004

Romer, C. D. (1990). The great crash and the onset of the Great Depression. Quarterly Journal of Economics, 105(3), 597-624. doi:10.2307/2937892 
Silvia, J., \& Iqbal, A. (2011). Monetary policy, fiscal policy, and confidence. International Journal of Economics and Finance, 3(4), 22-35.

Vieira, F. V., \& Da Silva, C. G. (2016). BRICS export performance: an ARDL bounds testing empirical investigation. $44^{\circ}$ Encontro Nacional de Economia Anpec, Foz do Iguaçu/PR. Disponível em: $<$ https://www.anpec.org.br/encontro/2016/ submissao/files_I/i7-80490f02382136018991d1557e20dde6.pdf> Acessado em: 20/05/2017

Vuchelen, J. (1995). Political events and consumer confidence in Belgium. Journal of Economic Psychology, 16(4), 563-579. doi:10.1016/0167-4870(95)000286

Vuchelen, J. (2004). Consumer sentiment and macroeconomic forecasts. Journal of Economic Psychology, 25(4), 493-506. doi:10.1016/s0167-4870(03)00031-x

\section{Apêndice}

Tabela 1A. Estatística Descritiva

\begin{tabular}{l|r|r|r|r|r}
\hline \hline \multicolumn{1}{c|}{ Variáveis } & \multicolumn{1}{c|}{ Média } & Mediana & \multicolumn{1}{c|}{ Máximo } & \multicolumn{1}{c}{ Mínimo } & \multicolumn{1}{c}{$\begin{array}{c}\text { Desvio } \\
\text { Padrão }\end{array}$} \\
\hline $\begin{array}{l}\text { Índice de C. do Consumidor } \\
\text { Índice de Preços ao }\end{array}$ & 100,91 & 101,00 & 102,85 & 97,84 & 1,27 \\
Consumidor & 99,732 & 94,775 & 149,43 & 65,261 & 23,173 \\
$\begin{array}{l}\text { Letras Financeiras do } \\
\text { Tesouro }\end{array}$ & $504.884,70$ & 519239,70 & 675662,20 & $308.576,30$ & $99.869,77$ \\
Índice de Atividade & 128,24 & 129,86 & 148,75 & 98,96 & 15,21 \\
Econômica & 13,95 & 13,55 & 20,61 & 9,92 & 3,14 \\
Taxa de Desemprego & $2.126,77$ & $2.111,74$ & $2.552,65$ & $1.687,17$ & 243,77 \\
Rendimento Médio Real & 13,10 & 11,93 & 26,32 & 7,11 & 4,36 \\
Taxa de Juros Selic & 82,14 & 84,77 & 109,76 & 46,30 & 15,69 \\
Taxa de Câmbio Real & & &
\end{tabular}

Fonte: Elaboração Própria 